\title{
Life history of Nippoleucon hinumensis (Crustacea: Cumacea: Leuconidae) in Seto Inland Sea of Japan. II. Non-diapausing subpopulation
}

\author{
T. Akiyama*, Masamichi Yamamoto \\ Ushimado Marine Laboratory, Okayama University, Ushimado, Okayama 701-4303, Japan
}

\begin{abstract}
The great majority of Nippoleucon hinumensis (Gamô) in the Seto Inland Sea of Japan enters a long-term summer diapause from May to November. We identified a minor subpopulation of $N$. hinumensis $(0.7 \%$ of the total population) that became adults in a short period without diapause. Small-sized ovigerous females (15\% shorter than the ordinary-sized ovigerous females) appeared from January to early February, a month earlier than the appearance of the ordinary-sized ovigerous females that produce diapausing offspring. These precociously ovigerous females released offspring from early February to early March. The offspring developed from 1st manca larvae to 2nd manca larvae, 1st juvenile stage and 2nd juveniles stage in that order, and in mid-April they reached the adult stage (5th instar) without diapause. In the non-diapausing subpopulation, sexual dimorphism was apparent from the 1st manca instar and the sex ratio (males:females) was 2 to $4: 1$. The molts, occurring at intervals of ca. $10 \mathrm{~d}$, were not highly synchronous among individuals. The non-diapausing females mostly disappeared in April without producing offspring, but many non-diapausing males survived until the ordinary-sized ovigerous females started incubation of the second brood in late April. Possibly, the non-diapausing subpopulation is maintained through mating of non-diapausing males with ordinary-sized ovigerous females. The non-diapausing mode of life in $N$. hinumensis may represent the ancestral life mode before this species acquired a summer diapause mechanism.
\end{abstract}

KEY WORDS: Crustacea $\cdot$ Cumacea $\cdot$ Leuconidae $\cdot$ Life history $\cdot$ Diapause

Resale or republication not permitted without written consent of the publisher

\section{INTRODUCTION}

Diapause, a seasonal cessation of development, is an important physiological process enabling animals to adapt to annual fluctuations in ambient temperatures or seasonal desiccation of the habitat. The life cycles of many arthropods include both diapausing and nondiapausing generations (Masaki 1983, Alekseev \& Fryer 1996), and comparisons of the 2 generations have highlighted the physiological mechanisms and ecological aspects of diapause.

In an earlier paper (Akiyama \& Yamamoto 2004, this volume), we reported that a population of Nippoleucon hinumensis (Gamô) (Crustacea: Cumacea) in the Seto Inland Sea of Japan undergoes a long-term summer diapause from May to November. Individuals born in early April begin fasting in the 2nd juvenile stage (4th instar) in mid-May, and remain in the same instar to the end of November.

We identified a very small subpopulation of Nippoleucon hinumensis that became adults in a short period without diapause. The non-diapausing individuals derived from small-sized ovigerous females that occurred about 1 mo earlier than the ordinary-sized females producing the diapausing offspring. In the present paper, we report the life history of this small subpopulation of non-diapausing $N$. hinumensis.

\section{MATERIALS AND METHODS}

Nippoleucon hinumensis (Gamô, 1967) (Crustacea: Cumacea: Leuconidae) collected in the central region of the Seto Inland Sea of Japan (off Ushimado, 


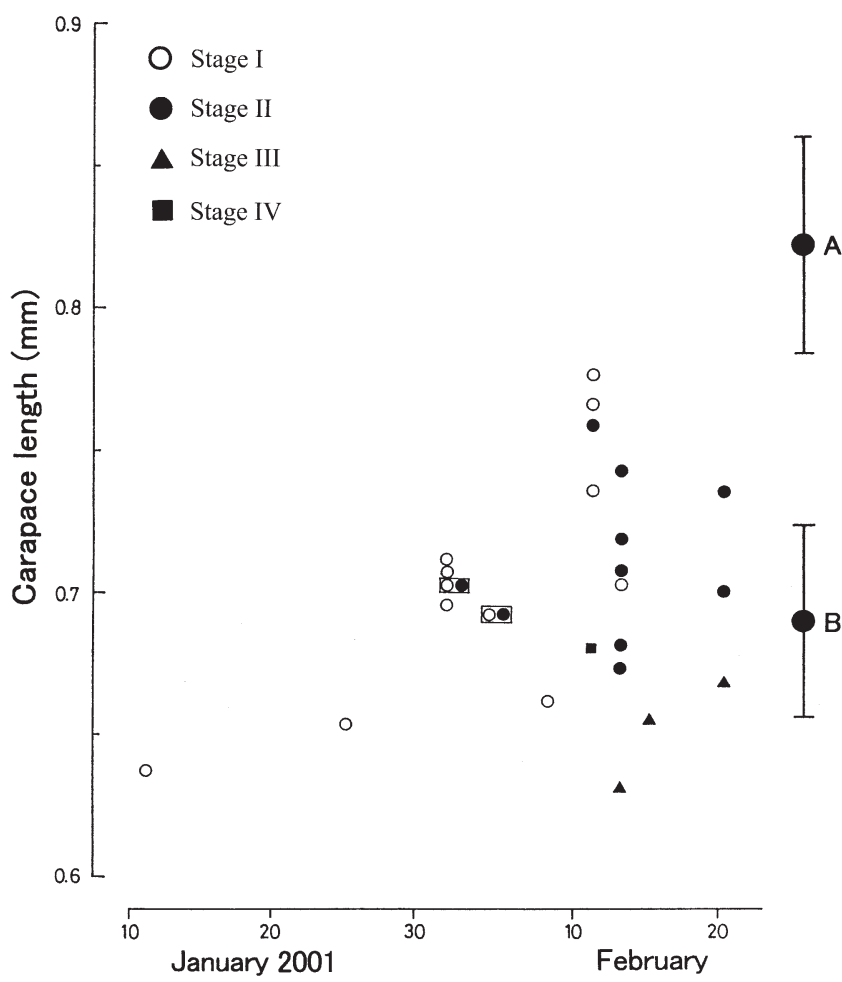

Fig. 1. Nippoleucon hinumensis. Carapace lengths of all females collected in 2001 and developmental stage of individuals incubated in their marsupiums. A: mean $( \pm S D, N=227)$ carapace length in ordinary-sized ovigerous females producing diapausing offspring; $\mathrm{B}$ : mean $( \pm \mathrm{SD}, \mathrm{N}=20)$ carapace length in precociously ovigerous females producing nondiapausing offspring

Okayama Prefecture; $\left.34^{\circ} 36^{\prime} \mathrm{N}, 134^{\circ} 09^{\prime} \mathrm{E}\right)$. Details of the sampling site and water temperature near the site have been described in Akiyama \& Yamamoto (2004).

The specimens described in the present paper and in Akiyama \& Yamamoto (2004) were sorted from the same sediment samples. Because of the scarcity of specimens of the non-diapausing subpopulation, sediment samples were gathered twice or thrice a week and at least 3 sediment samples were obtained on each sampling day from December 2000 to April 2001. To investigate the molt cycle of non-diapausing individuals, 15 to 20 sediment samples were collected at intervals of about $10 \mathrm{~d}$ from 23 February to 1 May 2001. For details of collection and treatment of the specimens see Akiyama \& Yamamoto (2004).

Carapace length was used as an index of body size. Length of the pseudorostrum was excluded from the carapace length (after Bishop 1982). Sex was determined on the basis of shape of the 4 th pereopod which, for all instars, developed an exopod only in males (see Fig. 5). Fecundity and intramarsupial stages were assessed by direct observation of the individuals re- moved from the marsupium. The intramarsupial stage was identical with that in Akiyama \& Yamamoto (2004). The postmarsupial stages were determined on the basis of the shape of the 4 th and 5th pereopods and the uropod in males, and the shape of the pereopod, uropod and oostegite plus body size in females.

\section{RESULTS}

\section{Precociously ovigerous females}

Relatively small-sized ovigerous females occurred in sediment samples from January to February, 1 mo earlier than the appearance of ordinary-sized ovigerous females that produce diapausing offspring as described in Akiyama \& Yamamoto (2004). We shall refer to the early-occurring, small, ovigerous females and the lateoccurring, ordinary-sized, ovigerous females as precociously ovigerous females (POF) and ordinary ovigerous females (OOF), respectively. As described in a later subsection, the POF produced offspring that showed no period of diapause. The POF were present in far fewer numbers than ordinary females: 24 POF specimens out of 13, 206 specimens of Nippoleucon hinumensis in 46 sediment samples from 12 January to 21 February 2001. The first POF specimen was collected on 12 January,

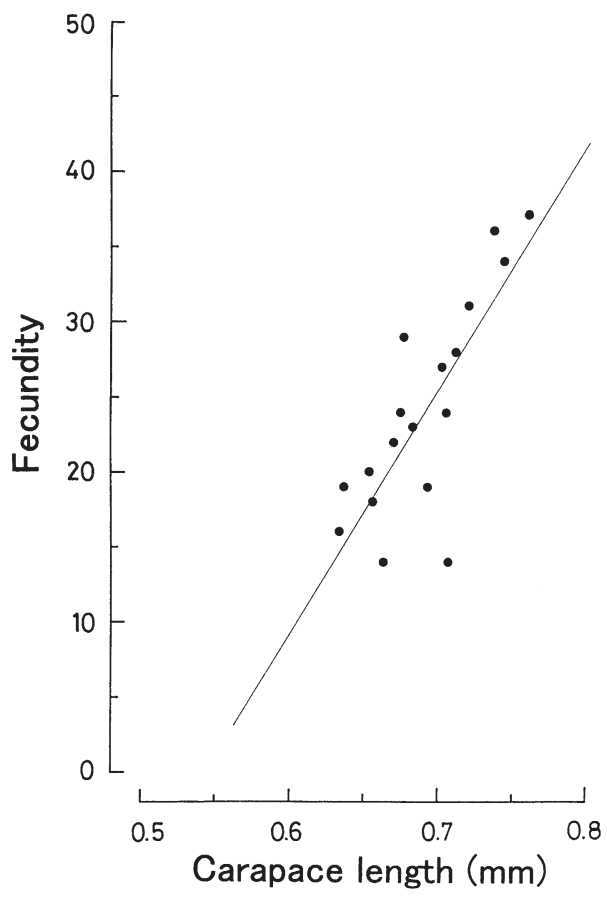

Fig. 2. Nippoleucon hinumensis. Fecundity (no. of individuals marsupium $^{-1}$ ) of precociously ovigerous females in relation to carapace length. Data from 18 of 24 specimens in Fig. 1 and linear regression line are shown 
the first 3 OOF specimens on 12 February. Fig. 1 shows the carapace lengths of all 24 specimens collected in 2001 and stages of the embryos in their marsupium. The carapace length of POF was $0.692 \pm 0.034 \mathrm{~mm}$, i.e. about $15 \%$ shorter than that of the OOF $(\mathrm{N}=20 ; 4$ specimens of incubating Stage I embryos after 10 February were excluded as they may have been OOF). The sex ratio (males:female) for the total specimens collected from 12 January to 21 February ranged from 1.5 to 2. The percentage of POF in the total females was thus estimated to be less than $0.5 \%$. The POF specimens were too few to determine the time-course of intramarsupial development. Mean fecundity (no. of ind. marsupium ${ }^{-1}$ ) of the POF was $24.2 \pm 7.2(\mathrm{~N}=18)$, which was less than half the fecundity for the first brood of the OOF. The regression line between fecundity $(y)$ and carapace length $(x, \mathrm{~mm})$ was $y=163.0 x-88.7$ (Fig. 2).

\section{1st manca larvae}

In 2001, the earliest 1st manca larva was obtained on 12 February. The top graph of Fig. 3 shows the temporal increase in the number of 1st manca larvae collected from late February through March. Since the individuals brooded by ordinary ovigerous females remained in early developmental stages from February to early March (Akiyama \& Yamamoto 2004), the 1st manca larvae collected in that period were regarded as the progeny of precociously ovigerous females. The number of 1 st manca larvae collected increased abruptly in late March, presumably as a result of release of larvae by OOF. The only criterion by which we could distinguish 1st manca larvae released by POF from those released by OOF was the sex ratio (males:females). Fig. 4 shows temporal fluctuations in the sex ratio of 1st manca larvae from late February through March. The sex ratio was greater than 2 from late February to mid-March, decreasing rapidly in late March to 0.85 , which corresponded to the sex ratio of 1st manca larvae released from OOF (Akiyama \& Yamamoto 2004). These results indicate that the 1st manca larvae released by OOF replaced those released by POF in mid-March. The carapace length of 1 st manca larvae deriving from POF was ca. $6 \%$ greater than that of 1st manca larvae from ordinary females.

\section{Postmarsupial development without diapause}

The offspring of the POF were morphologically distinct from those of ordinary ovigerous females at the 2nd manca instar. In males, the exopod on the basal segment of the 4 th pereopod increased in length with each molt (Fig. 5) in the POF offspring, while it remained short until the end of the summer diapause in the offspring of ordinary females (Akiyama \& Yamamoto 2004). In the females, difference in body
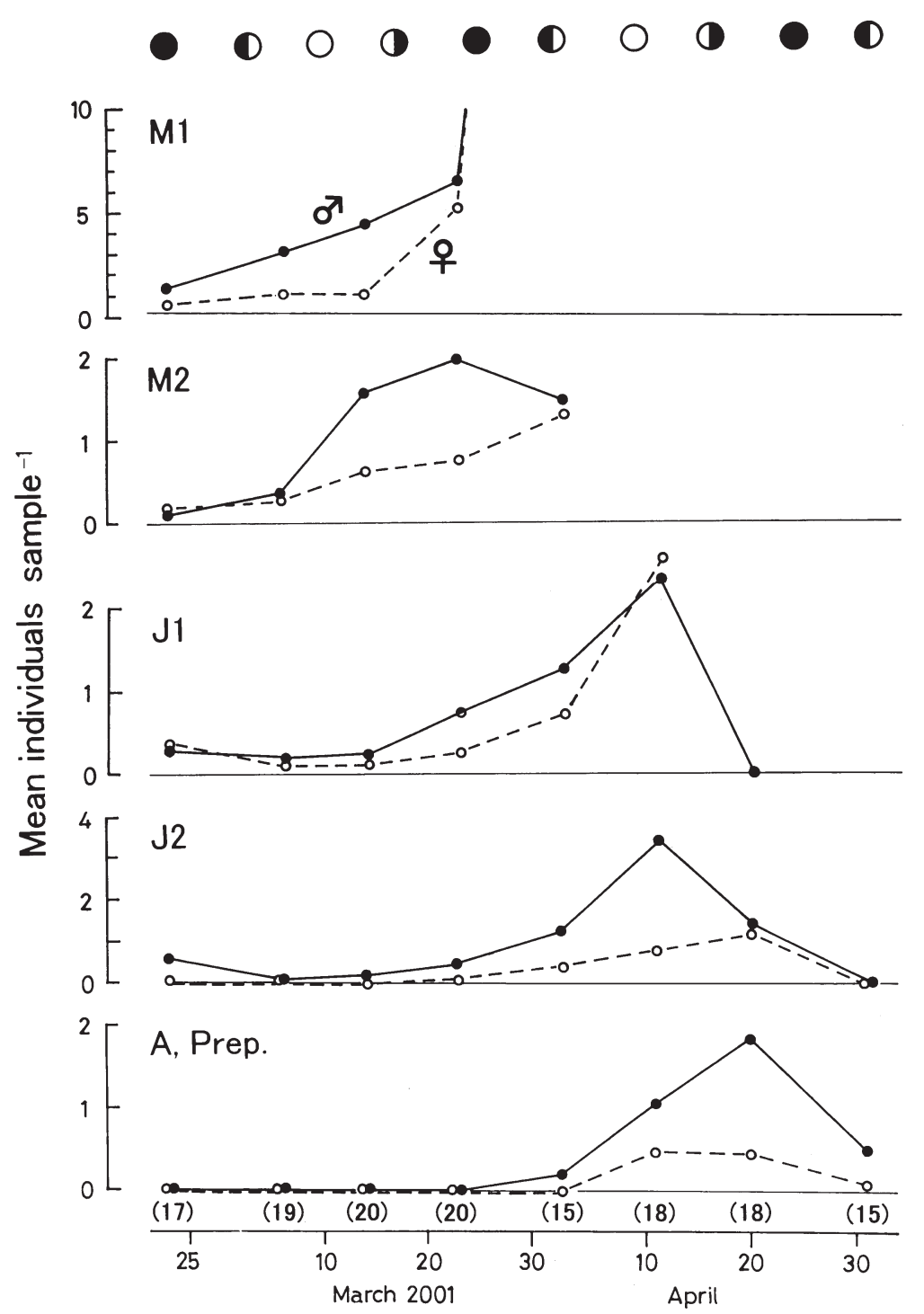

Fig. 3. Nippoleucon hinumensis. Temporal change in abundance of nondiapausing individuals at each instar in 2001. Ordinate shows mean number of specimens per sediment sample; number of sediment samples examined each day is indicated in parentheses on abscissa. M1, M2: 1st and 2nd manca, respectively; J1, J2: 1st and 2nd juveniles stages, respectively; A: adult male; Prep: preparatory female. Phases of the moon are shown at the top: $(\mathbf{\bullet})$ new moon; ( $\mathbf{O})$ first quarter; (O) full moon; $(\mathbf{O})$ last quarter 


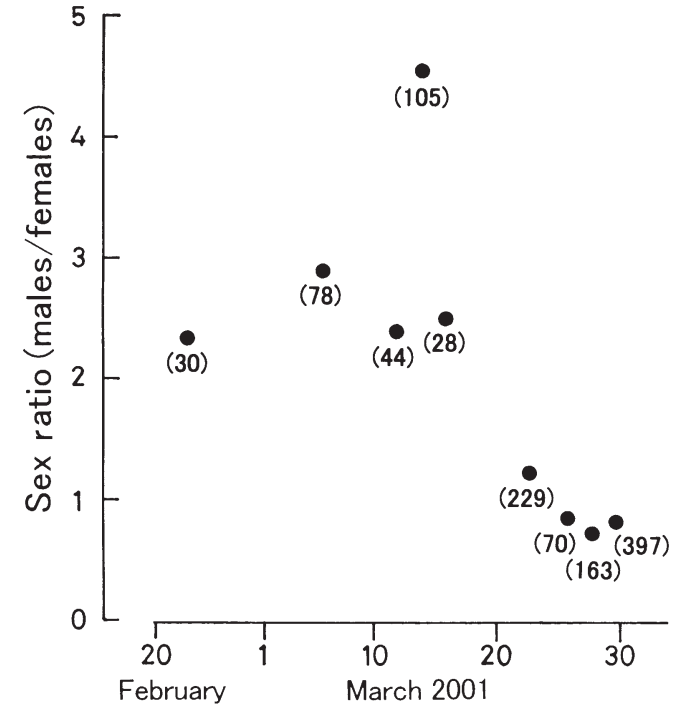

Fig. 4. Nippoleucon hinumensis. Sex ratio of 1st manca larvae released from 23 February to 30 March 2001. Number of specimens examined indicated in parentheses

length was a good criterion for discriminating POF

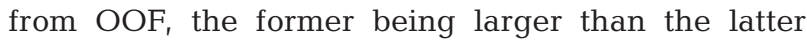
(Fig. 6). Continual sampling revealed that POF offspring reached the adult stage (adult male or preparatory female) through 4 instars (1st manca, 2nd manca, 1st juvenile and 2nd juvenile) in mid-April (Fig. 3). Morphological details of each instar will be reported elsewhere. In contrast to OOF offspring, which undergo a long diapause during postmarsupial development (Akiyama \& Yamamoto 2004), POF offspring displayed
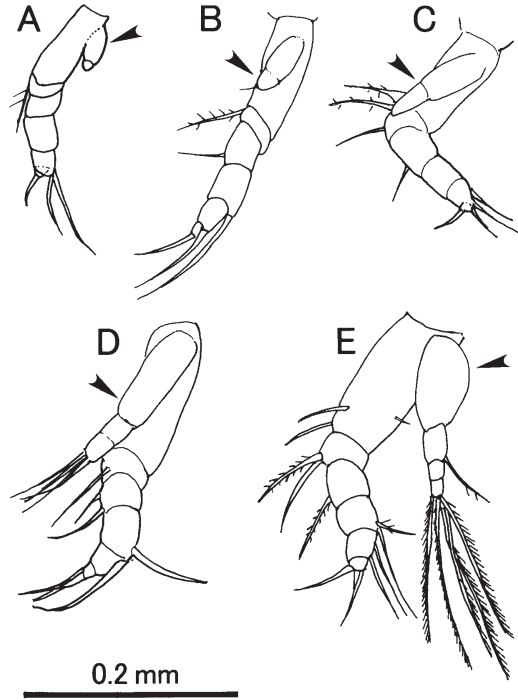

Fig. 5. Nippoleucon hinumensis. Development of 4th pereopod in non-diapausing males. (A) 1st manca; (B) 2nd manca; (C) 1st juvenile instar; (D) 2nd juvenile instar; (E) adult male. Arrowhead indicates exopod

gradual growth with no diapause (Fig. 6). Hereafter, we shall refer to OOF and POF offspring as diapausing and non-diapausing individuals, respectively. The postmarsupial period of non-diapausing individuals was much shorter than that of diapausing individuals: it consisted of 3 fewer instars (Fig. 6), and did not include a diapause. Diapausing individuals store numerous lipid droplets before entering the diapause (Akiyama \& Yamamoto 2004), but only a few lipid droplets were observed in the dorsal part of the cara-
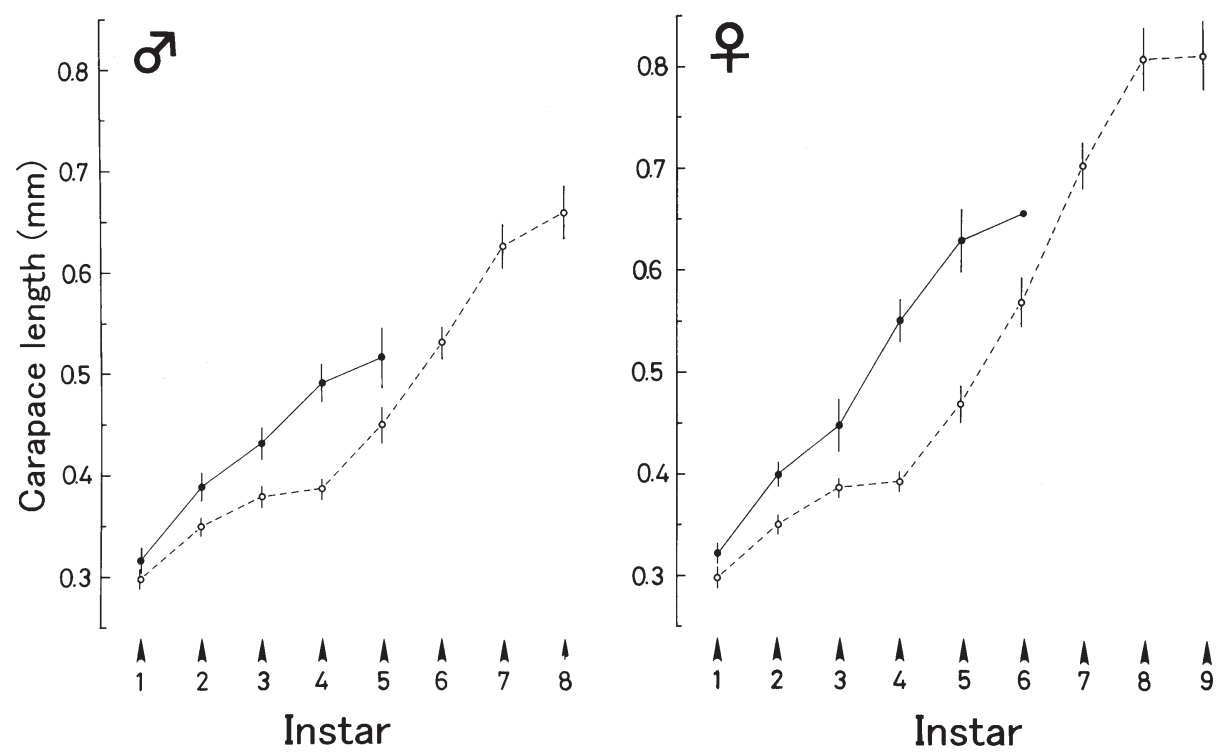

Fig. 6. Nippoleucon hinumensis. Comparison of carapace length between diapausing (dashed lines) and non-diapausing (continuous lines) individuals. In males, last instar in both diapausing and nondiapausing individuals is adult; in females, 8th instar in diapausing individuals and 5th instar in nondiapausing individuals are preparatory females 
pace and the free thoracic segments in the 2nd juvenile of non-diapausing individuals.

\section{Growth}

Observations over 3 yr revealed that for each instar, mean carapace length decreased over the period each year that it was present in the population (Fig. 7). We regarded the average carapace length on the day on which population density reached a maximum for each instar as the representative carapace length for that instar. Table 1 shows carapace lengths of nondiapausing individuals. Percentage growth at first molt $(22.7 \%$ for males, $24.2 \%$ for females) was greatest throughout the postmarsupial period in both diapausing and non-diapausing individuals. Growth rate was relatively slower at the second molt, and increased again at the third molt. It was very slow at the last molt, possibly as a result of diversion of resources to spermatogenesis and vitellogenesis. Percentage growth in females was higher than that in males at each molt, and as a result ovigerous females were ca. $25 \%$ longer than adult males. Fig. 6 compares growth between diapausing and non-diapausing individuals. Nondiapausing individuals were larger than diapausing individuals at each instar up to the adult stage in both sexes. Since non-diapausing individuals reached the adult stage after 3 fewer molts than diapausing individuals, the adult males and preparatory females of non-diapausing individuals were much smaller than those of diapausing individuals (Fig. 6).

\section{Molt cycle}

The shift in abundance of non-diapausing individuals at each instar (Fig. 3) revealed their molt cycles. Temporal changes in abundance of non-diapausing individuals in 1999 and 2000 (Fig. 8) were similar to those in 2001 (Fig. 3), although quantitative data on the 1st manca larvae were not obtained in 1999 and 2000 because of the large mesh size of the sampling gear used. Male and female abundance appeared almost synchronous at each instar (Fig. 3). The intervals of peak abundance between instars showed that molts occurred at intervals of about $10 \mathrm{~d}$ following emergence of the 1st manca larvae in the first half of March. The broad peaks in the temporal pattern of abundance indicated that each molt was not strongly synchronous among non-diapausing individuals. Synchronization of the molt cycle with the phase of the moon, as observed in diapausing individuals after the summer diapause (Akiyama \& Yamamoto 2004) was not evident in nondiapausing individuals.

\section{Abundance}

Population density of non-diapausing individuals was far lower than that of diapausing individuals. The number of specimens of the non-diapausing individuals was 2.3 per sediment sample on 20 April 2001, when the abundance of adult males and preparatory females reached a maximum (Fig. 3). The number of specimens of diapausing individuals was $311.3 \pm 278.7$

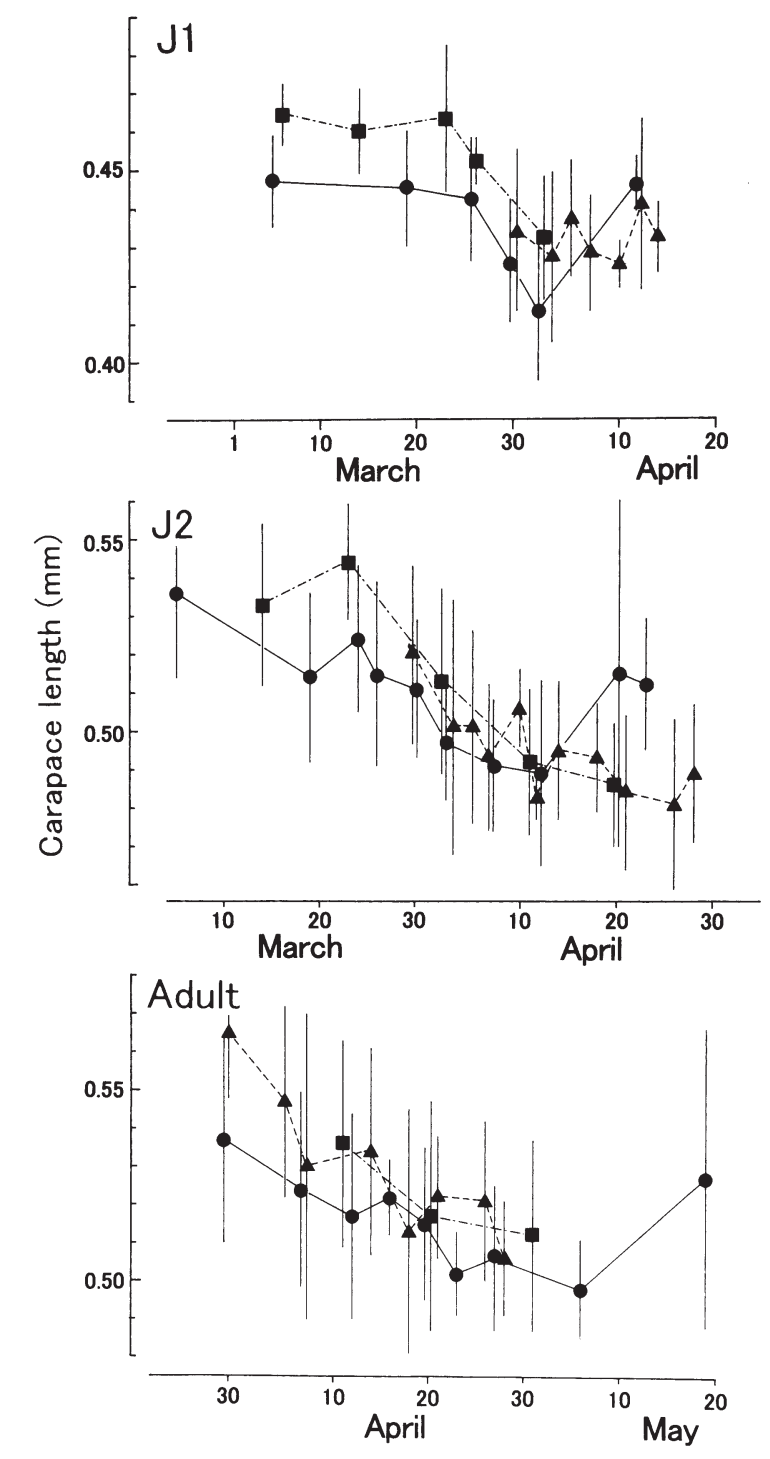

Fig. 7. Nippoleucon hinumensis. Changes in carapace length during the instar of 1st juvenile (J1), 2nd juvenile (J2), and adult male (adult). Mean $( \pm \mathrm{SD})$ carapace lengths measured in specimens collected in (•) 1999, (४) 2000, and (•) 2001. Mean $( \pm$ SD) numbers of specimens examined each a day was $5.8 \pm$ 2.3 (1999), $7.0 \pm 4.9(2000)$ and $8.6 \pm 6.9$ (2001) for the 1 st juvenile, $7.1 \pm 3.3(1999), 9.9 \pm 5.8(2000)$ and $13.5 \pm 7.5$ (2001) for the 2nd juvenile, and $8.8 \pm 4.3$ (1999), $9.5 \pm 5.1$ (2000) and $17.7 \pm 7.1(2001)$ for the adult 
Table 1. Nippoleucon hinumensis. Growth of non-diapausing individuals. Sampling dates: 1st manca, 6 March; 2nd manca, 14 March; 1st juvenile instar, 2 April; 2nd juvenile instar, 11 April; adult male and preparatory females, 20 April; ovigerous female, 1 May. Sampling year 2001. N: number of specimens. Growth (\%): percentage increase from preceding stage

\begin{tabular}{|c|c|c|c|c|}
\hline \multirow{2}{*}{ Instar } & \multicolumn{2}{|l|}{ Males - } & \multirow{2}{*}{$\begin{array}{l}\text { Carapace length }(\mathrm{mm}) \\
\text { Females } \\
\text { (mean } \pm \mathrm{SD})\end{array}$} & \multirow[b]{2}{*}{$\begin{array}{c}\text { Growth } \\
(\%)\end{array}$} \\
\hline & $\begin{array}{l}\text { Carapace length }(\mathrm{mm}) \\
\quad(\text { mean } \pm \text { SD })\end{array}$ & $\begin{array}{c}\text { Growth } \\
(\%)\end{array}$ & & \\
\hline 1st manca & $0.317 \pm 0.013(\mathrm{~N}=20)$ & \multirow{5}{*}{$\begin{array}{c}22.7 \\
11.1 \\
13.9 \\
5.1\end{array}$} & $0.322 \pm 0.010(\mathrm{~N}=15)$ & \multirow{5}{*}{$\begin{array}{l}24.2 \\
12.0 \\
23.0 \\
14.2\end{array}$} \\
\hline 2nd manca & $0.389 \pm 0.014(\mathrm{~N}=20)$ & & $0.400 \pm 0.012(\mathrm{~N}=16)$ & \\
\hline 1st juvenile & $0.432 \pm 0.016(\mathrm{~N}=19)$ & & $0.448 \pm 0.026(\mathrm{~N}=7)$ & \\
\hline 2nd juvenile & $0.492 \pm 0.019(\mathrm{~N}=21)$ & & $0.551 \pm 0.021(\mathrm{~N}=33)$ & \\
\hline Adult male/ & $0.517 \pm 0.030(\mathrm{~N}=25)$ & & $0.629 \pm 0.031(\mathrm{~N}=8)$ & \\
\hline Preparatory female & & & & \multirow[t]{2}{*}{4.1} \\
\hline Ovigerous female & - & - & $0.655(\mathrm{~N}=2)$ & \\
\hline
\end{tabular}

$(\mathrm{N}=30)$ per sediment sample from 29 January to 21 February 2001, when most of the diapausing individuals were at the adult stage (adult males or preparatory females). Thus the relative abundance of non-diapausing to diapausing individuals was estimated to be $0.7 \%$. At all instars of non-diapausing individuals, males were more frequent than females (Fig. 3). The number of 1st manca larvae of the nondiapausing subpopulation collected in early March 2001 was 4.1 per sediment sample. The number of adult males and preparatory females of the non-dia- pausing subpopulation collected in mid-April was 2.3 per sediment sample. It was estimated from these values that about half the non-diapausing individuals survived to the adult stage. Ovigerous females of the non-diapausing subpopulation were rarely encountered in the sediment samples: we obtained only 2 specimens in early May 2001. It seems from these results that most females of the non-diapausing subpopulation died during the preparatory female instar. One of the non-diapausing ovigerous females contained 14 embryos in its marsupium, a much lower
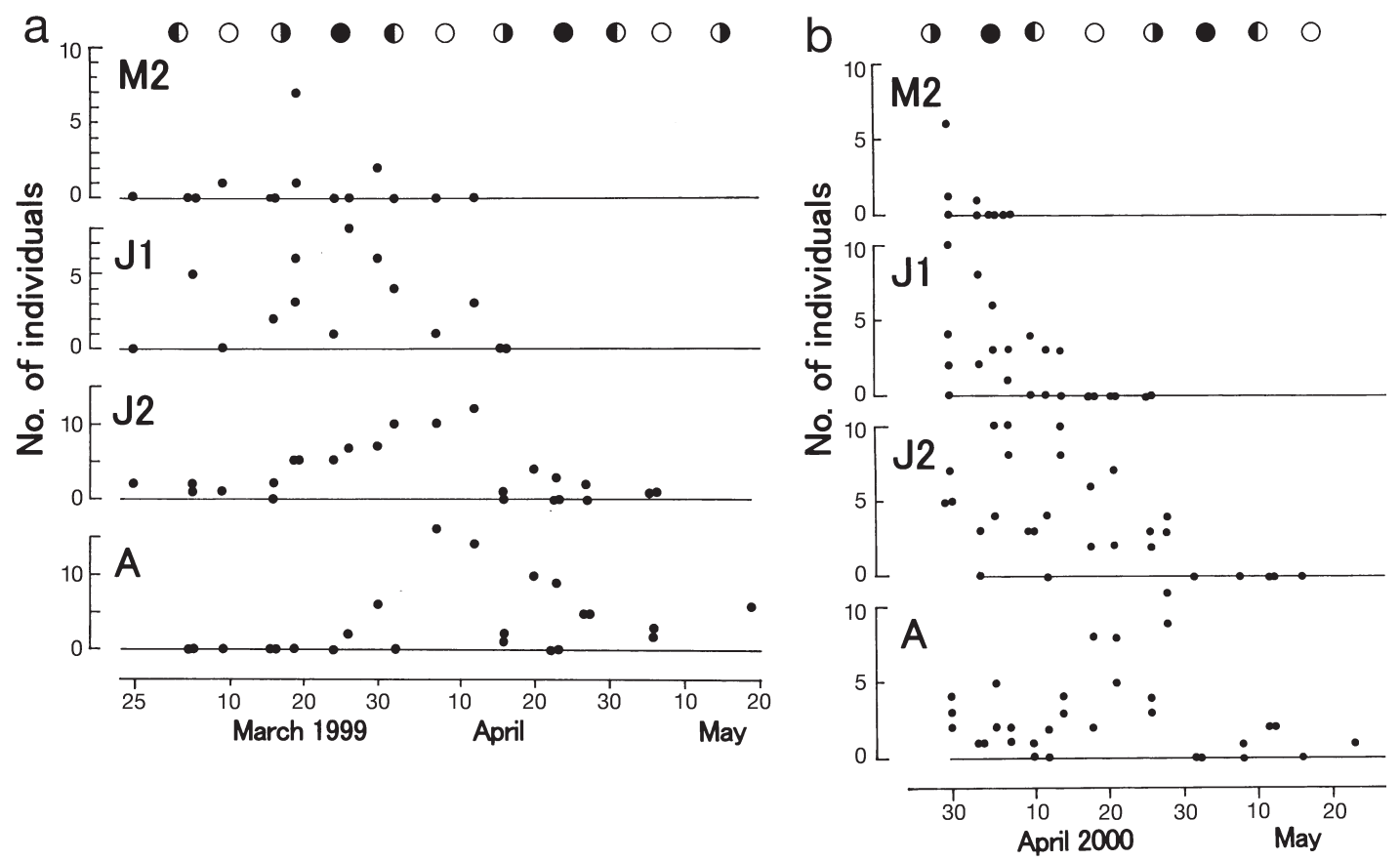

Fig. 8. Nippoleucon hinumensis. Temporal change in abundance of non-diapausing individuals at each instar in (a) 1999 and (b) 2000. Each data point indicates number of specimens collected by 1 tow of epibenthic sledge; other symbols and abbreviations as in Fig. 3 
number than the 30 to 80 usually incubated by diapausing ovigerous females. We have not yet detected larvae released by non-diapausing females in sediment samples. Males of the non-diapausing subpopulation survived until after the non-diapausing females had mostly disappeared. Since the males of the diapausing subpopulation abruptly disappeared when the ordinary ovigerous females started to incubate their first brood (Akiyama \& Yamamoto 2004), the relative abundance of non-diapausing to diapausing males rapidly increased from $<1$ to $\sim 100 \%$ during April (Fig. 9).

\section{DISCUSSION}

\section{Diapausing and non-diapausing individuals}

The life history of Nippoleucon hinumensis in the Seto Inland Sea of Japan is summarized in Fig. 10. Offsprings of the precociously ovigerous females emerged shortly before initiation of the first brood in ordinary females, and became adults without a diapause shortly before initiation of the second brood of the ordinary females. Thus there were 2 subpopulations of N. hinumensis, diapausing and non-diapausing, in the study area from at least March through May. Non-diapausing individuals differ from diapausing individuals as follows: (1) shorter life span; (2) 3 fewer molts; (3) faster succession of instars; (4) weaker synchronization of each molt among individuals; (5) smaller adult body size; (6) no delay in development of male sexual characteristic; (7) marked predominance of males; (8) paucity of lipid droplets in the body cavity.

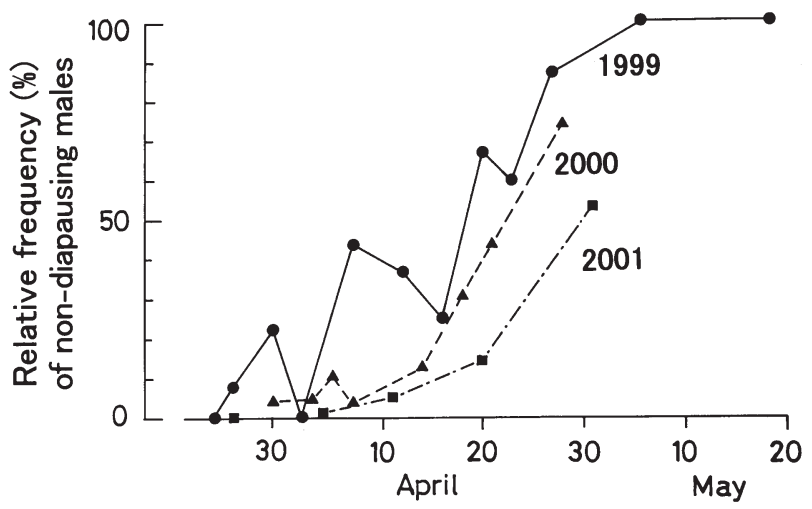

Fig. 9. Nippoleucon hinumensis. Changes in relative abundance of non-diapausing adult males to diapausing adult males following release of manca larvae from first brood of ordinary-sized females in $(\bullet)$ 1999, (ム) 2000, and (Ш) 2001

\section{Life history of non-diapausing individuals}

Since precociously ovigerous females and their non-diapausing offspring were very few in number, a complete description of the life history of non-diapausing individuals is difficult. Although the origin of precociously ovigerous females is not known, smallsized individuals that comprise less than $1 \%$ of the population during the summer diapause (Akiyama \& Yamamoto 2004) are conceivable candidates. Since we did not detect manca larvae released from nondiapausing offspring of the precociously ovigerous females, it is not certain whether the life cycle of the non-diapausing subpopulation is continued by their offspring. Since non-diapausing females at the
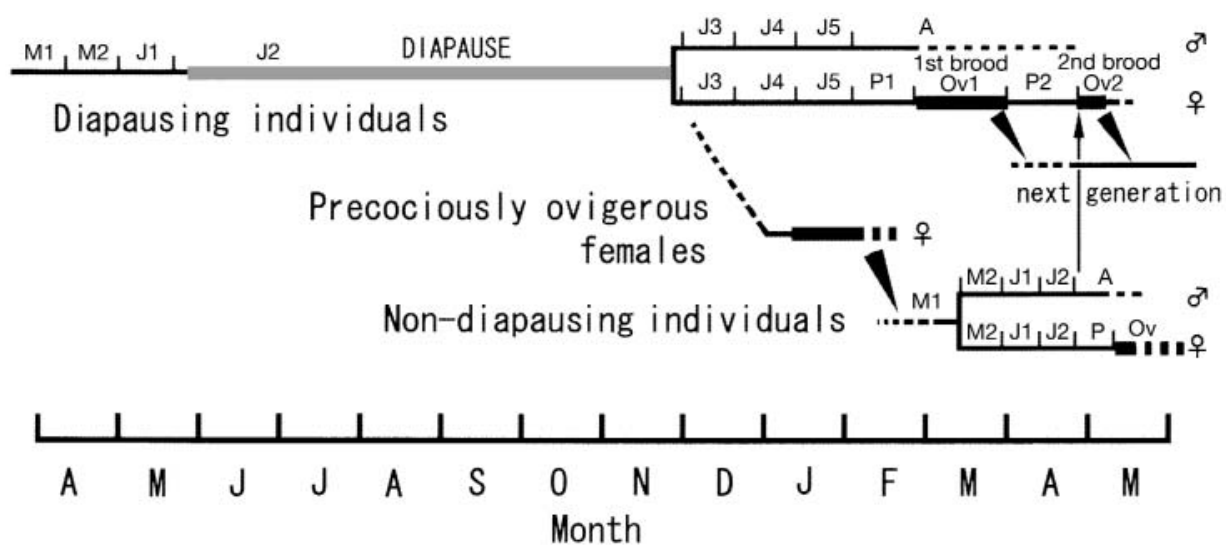

Fig. 10. Nippoleucon hinumensis. Summary of life histories of diapausing and non-diapausing subpopulations in the Seto Inland Sea of Japan. Downward arrowheads indicate release of manca larvae; arrow from adult male (A) of non-diapausing individuals to 2nd ovigerous females of diapausing individuals shows presumed mating; oblique broken line represents presumption that precociously ovigerous females originate from diapausing individuals. M1, M2: 1st and 2nd manca, respectively; J1-J5: 1st-5th juvenile, respectively; P: preparatory female; P1, P2: 1st and 2nd preparatory females; Ov: ovigerous female; Ov1, Ov2: 1st and 2nd ovigerous females, respectively 
ovigerous stage were very rare, it would seem that most die before brooding. If no offspring are released from non-diapausing females, then non-diapausing males may play a role in the permanence of a nondiapausing subpopulation of Nippoleucon hinumensis. Since many diapausing males died immediately after mating with ordinary females before the first brood (Akiyama \& Yamamoto 2004), non-diapausing males were about 20 to $60 \%$ as abundant as diapausing males when the ordinary ovigerous females began their second broods in late April (Fig. 9). Nondiapausing males may be involved in the production of offspring from the second brood through mating with ordinary ovigerous females. In our previous paper (Akiyama \& Yamamoto 2004), we postulated that during the summer diapause, the offspring from the second brood form a minor group of 1st stage juveniles that is distinct from the major group of 2 nd stage juveniles from the first brood. If this is the case, the non-diapausing generation of $N$. hinumensis alternates with the diapausing generation in an unusual way, comparable with the typical alteration of diapausing and non-diapausing generations reported for many insects (Masaki 1983). Direct observstion of the reproductive behavior of nondiapausing males in the laboratory is required.

\section{Factors controlling diapause}

It is not certain what controls the diapausing or nondiapausing mode of life in Nippoleucon hinumensis. Since females producing non-diapausing offspring are distinguishable from females producing diapausing offspring by their smaller size, the life mode of the offspring may be genetically determined. In insects, seasonal fluctuations in day length and the ambient temperature have been reported as effective stimuli inducing a diapause (Saunders 1982). If the life mode of $N$. hinumensis is environmentally controlled, then both changes in seawater temperature and day length could be candidates as stimuli controlling the diapause. Since diapausing and non-diapausing individuals differ as early as at the 1st manca stage in such characters as carapace length and the accumulation of lipid droplets, environmental factors would have to affect embryos brooded in the marsupium. During intramarsupial development of non-diapausing individuals brooded by precociously ovigerous females (mid-January to early March), seawater temperature decreased from $9.8 \pm 0.7$ to $8.2 \pm 0.4^{\circ} \mathrm{C}$, and daylight lengthened from $10 \mathrm{~h} 5 \mathrm{~min}$ to $11 \mathrm{~h} 34 \mathrm{~min}$. During intramarsupial development of diapausing individuals brooded by ordinary ovigerous females (late February to early April), the seawater temperature increased from $8.3 \pm 0.6$ to $10.7 \pm 0.8^{\circ} \mathrm{C}$, and daylight lengthened from $11 \mathrm{~h} 16 \mathrm{~min}$ to $12 \mathrm{~h} 41 \mathrm{~min}$. To clarify the mechanism controlling the diapause in $N$. hinumensis, experimental analyses as well as comparison of the life history of local populations in various climatic regions will be necessary.

\section{Evolutionary significance}

We think that the non-diapausing way of life may be a relic from the life mode of the ancestors of Nippoleucon hinumensis, before acquisition of a physiological mechanism for the summer diapause. As discussed in Akiyama \& Yamamoto (2004), the summer diapause in $N$. hinumensis seems to be a result of adaptation to increased water temperature accompanying the southern expansion of the originally coldwater species. A diapause has not been reported in the life history of leuconid cumaceans living in cold waters of the shallow (Granger et al. 1979) or deep (Bishop 1982, Bishop \& Shalla 1994) sea. Progressive growth of the exopod of the 4th pereopods during postmarsupial development as observed in the nondiapausing male $N$. hinumensis seems to be a common character of the Leuconidae (Forsman 1938, Bishop 1982). In diapausing N. hinumensis, however, growth of the exopod is retarded during the first 3 molts before the summer diapause. The highly synchronous molts among individuals observed in the diapausing subpopulation of $N$. hinumensis have also not been reported in other leuconid cumaceans. Thus, the highly synchronous molts and retarded growth of the exopod seem to be characters derived in parallel with the acquisition of the mechanism of summer diapause in $N$. hinumensis.

Because of their very low population density, nondiapausing individuals do not seem to play a significant role in the maintenance of the local population of Nippoleucon hinumensis in the Seto Inland Sea of Japan. However, in local populations living in habitats at lower ambient temperatures, non-diapausing individuals may have a suitable niche. Unfortunately, there have been no studies on the geographic distribution of Nippoleucon species along the coasts of Japan and vicinity. To understand the evolutionary significance of various modes in the life history of the Leuconidae, we have begun comparative studies among many leuconid species from different climatic regions.

Acknowledgements. We thank Mr. W. Godo of Ushimado Marine Laboratory for technical assistance. Thanks are also due to Professor Emeritus S. Gamô of Yokohama National University for identification of the specimens. We also thank Dr. I. Gleadall for improvement of the English manuscript. 


\section{LITERATURE CITED}

Akiyama T, Yamamoto M (2004) Life history of Nippoleucon hinumensis (Crustacea: Cumacea: Leuconidae) in Seto Inland Sea of Japan. I. Summer diapause and molt cycle. Mar Ecol Prog Ser 284:211-225

Alekseev V, Freyer G (eds) (1996) Diapause in Crustacea. Dev Hydrobiol 114:1-241

Bishop JDD (1982) The growth, development and reproduction of a deep sea cumacean (Crustacea: Peracarida). Zool J Linn Soc 74:359-380

Bishop JDD, Shalla SH (1994) Discrete seasonal reproduction in an abyssal peracarid crustacean. Deep-Sea Res 41:

Editorial responsibility: Otto Kinne (Editor),

Oldendorf/Luhe, Germany
$1789-1800$

Forsman B (1938) Untersuchungen über die Cumaceen des Skageraks. Zool Bidr Upps 18:1-161

Gamô S (1967) Studies on the Cumacea (Crustacea, Malacostraca) of Japan. Part I. Publ Seto Mar Biol Lab 15:133-163

Granger D, Brunel P, Messier D (1979) Cycle de développement de Leucon nasica (Crustacea, Cumacea) dans la nappe glaciale circalittorale de la baie des Chaleurs, golfe du Saint-Laurent, en 1968 et 1969. Can J Zool 57:95-106 Masaki S (1983) Summer diapause. Annu Rev Entomol 25: $1-25$

Saunders DS (1982) Insect clocks, 2nd edn. Pergamon Press, Oxford

Submitted: January 9, 2004; Accepted: July 15, 2004

Proofs received from author(s): December 1, 2004 